\title{
Electrical stimuli improve osteogenic differentiation mediated by aniline pentamer and PLGA nanocomposites
}

\author{
JIAN CAO $^{1,2^{*}}$, YUHONG MAN ${ }^{1 *}$ and LISEN LI ${ }^{1}$ \\ ${ }^{1}$ Department of Hand Surgery, China-Japan Union Hospital of Jilin University, Changchun, Jilin 130033; \\ ${ }^{2}$ Department of Orthopedics, Chifeng Municipal Hospital, Chifeng 024000, P.R. China
}

Received November 14, 2012; Accepted February 6, 2013

DOI: $10.3892 / b r .2013 .70$

\begin{abstract}
Electrical stimulation may improve the proliferation of animal cells. In the present study, osteoblasts were cultured on electroactive aniline pentamer (AP)/poly(lactic-coglycolic acid) (PLGA) copolymer composites, on which electric pulse was imposed. The combination of polymer and electric pulse enhanced the osteogenic differentiation of the osteoblasts, characterized by the upregulated expression of bone morphogenetic protein (BMP)-2, collagen I and osteonectin and the phosphorylation of Samd4, in contrast to polymer or electrical pulse alone. This action occurred in a polymer content-dependent manner. Therefore, the action of the electric pulse, assisted by the electroactive polymer implant, may be promising in the expedition of injured bone repair.
\end{abstract}

\section{Introduction}

Electric field pulse may improve the proliferation and differentiation of osteoblasts and the rehabilitation of injured bones. In the treatment of various bone injuries, electrical stimuli promote callusogenesis and play an important role in the regulation of the adhesion, proliferation and differentiation of osteoblasts (1-3). Even very small electrical stimuli may trigger DNA synthesis and cell division, which is attributed to the bioelectrical nature of cells (4).

Bone repair materials have been found to interact with osteoblasts and promote cell proliferation and osteogenic differentiation (5-7). Currently, there is increasing interest in conducting polymers with unique chemical and physical properties. Several conducting polymers have been used to create biosensors, neural probes, drug release regulators, initiators and auto-oxidants (8-10). Conducting polymers

Correspondence to: Dr Lisen Li, Department of Hand Surgery, China-Japan Union Hospital of Jilin University, 126 Xiantai Street, Changchun, Jilin 130033, P.R. China

E-mail: leelisen@163.com

*Contributed equally

Key words: osteogenesis, osteoblasts, nanocomposite, electric field are also preferred for biomedical applications, due to their beneficial bone-healing properties (11-13). Wong et al (11) confirmed that conducting polymers effectively control the shape and growth of mammalian cells. Polyanilines (PAs) are a class of conducting polymers which appear promising, due to their stability and easy synthesis. Aniline oligomer composited with aliphatic polyester possesses conductive and biodegradable-biocompatible properties. The use of aniline oligomer/aliphatic polyester composites appears promising in the enhancement of the effect of electric pulse on the expedition of injured bone healing by multiple mechanisms.

In the present study, external electric pulse was imposed on osteoblasts, which were cultured on nanocomposites created from aniline oligomers and poly(lactic-co-glycolic acid) (PLGA) blends. We investigated the adhesion, proliferation and differentiation of osteoblasts under the action of conducting polymers in combination with electrical stimuli.

\section{Materials and methods}

Preparation and characterization of AP/PLGA nanocomposites. Preparations of aniline pentamer (AP) and its chemical structures have been previously described $(12,13)$. AP and PLGA were dissolved in chloroform to form a $0.06 \mathrm{~g} / \mathrm{ml}$ solution. First they were mixed together and then stirred and sonicated. Solid nanocomposites were obtained by precipitation in anhydrous alcohol and were dried in vacuum for $48 \mathrm{~h}$. The nanocomposites were characterized as previously described $(12,13)$.

Cell seeding. The nanocomposites and other materials were dissolved in chloroform $(0.1 \mathrm{~g} / \mathrm{ml})$ to form a $10 \mathrm{wt} \%$ solution. The solution $(0.5 \mathrm{ml})$ was dropped onto a $15-\mathrm{mm}$ Fisherbrand coverslip (treated with $2 \%$ dimethyldichlorosilane/chloroform solution, then dried at $180^{\circ} \mathrm{C}$ for $4 \mathrm{~h}$ prior to use) and evaporated in vacuum for $48 \mathrm{~h}$ at room temperature to form a thin film. Prior to use, the coverslips were sterilized by UV illumination for $40 \mathrm{~min}$.

Rat osteoblasts were freshly isolated in the laboratory as previously described (2) and seeded with a density of $5.0 \times 10^{4}$ cells/well $(1 \mathrm{ml}$ medium per well) in 6 -well plates (Corninng Costar) containing the coated coverslips. Cells were cultured in DMEM (Gibco, Carlsbad, CA, USA) supplemented with $10 \%$ fetal bovine serum (Gibco), $1.0 \times 10^{5} \mathrm{U} / 1$ penicillin 
Table I. Gene primer sequences.

\begin{tabular}{ll}
\hline Gene & \multicolumn{1}{c}{ Primer sequences } \\
\hline BMP-2 & F: 5'-GCAAGGTGTCTCCA-3' \\
Collagen I & R: 5'-CGCTGTTTGTGTTTC-3' \\
& F: 5'-TCGCTCACCACCTTCTC-3' \\
Osteonectin & R:5'-TAACCACTGCTCCACTCT-3' \\
& F: 5'-CGAAGAGGAAGTAGTG-3' \\
GAPDH & R: 5'-GAAGTGGCAGGAGGA-3' \\
& F: 5'-GATGGTGAAGGTCGGA-3' \\
& R: 5'-GTGGAGGTCAATGAAT-3' \\
\hline
\end{tabular}

BMP-2, bone morphogenetic protein-2; GAPDH, glyceraldehyde3-phosphate dehydrogenase.

(Sigma-Aldrich, St. Louis, MO, USA) and $100 \mathrm{mg} / \mathrm{l}$ streptomycin (Sigma-Aldrich), at $37^{\circ} \mathrm{C}$, in a humidified incubator with $5 \% \mathrm{CO}_{2}$.

Electrical stimuli. The electrical stimuli were initiated $24 \mathrm{~h}$ after cell seeding. Each well of the 6-well plate had a pair of platinum electrodes (anode and cathode) placed in the cover. Function signal generator (TFG6030 DDS Function Signal Generator, China) supplied the electrical stimuli with a voltage of $2 \mathrm{~V}$ and a frequency of $100 \mathrm{~Hz}$ for $1 \mathrm{~h}$ per day. The stimulus parameters were detected by a Rigol DS1022C Digital Oscilloscope (Rigol, Guangdong, China). The experiments included five groups: AP/PLGA-stimulated (0.1, 1 and 5\% AP), AP/PLGA-unstimulated and PLGA-stimulated.

Detections. Following electrical stimulation, the methyl thiazolyl tetrazolium (MTT; Sigma-Aldrich) method was used to assess cell viability (14). The absorbance was measured at $492 \mathrm{~nm}$ with a fully-automatic Microplate Reader (Biotek, Seattle, WA, USA).

The osteogenesis-associated genes, including collagen I, bone morphogenetic protein (BMP)-2 and osteonectin, were detected by quantitative real-time polymerase chain reaction (qPCR) (15). The RNA extraction kit and reverse transcription reagents were purchased from Promega (Madison, WI, USA); the qPCR kit was purchased from Stratagene (La Jolla, CA, USA). Primers were designed based on GenBank reference sequences and were verified by BLAST. Primer sequences are listed in Table I. The qPCR amplification program included predenaturation at $95^{\circ} \mathrm{C}$ for 2 min, denaturation at $95^{\circ} \mathrm{C}$ for $10 \mathrm{sec}$, annealing at $54^{\circ} \mathrm{C}$ for $20 \mathrm{sec}$ and extension at $72^{\circ} \mathrm{C}$ for $10 \mathrm{sec}$. Data analysis was performed using MxPro software equipped with Real-Time PCR Amplifier (Stratagene). GAPDH was used as the internal control.

Cells were lysed in ice-cold radioimmunoprecipitation assay lysis buffer (Beyotime, Nanjing, China) for $15 \mathrm{~min}$. Proteins were separated on 10\% SDS-PAGE gels and electroblotted onto a polyvinylidene fluoride (PDVF) film. The PDVF film was incubated overnight at $4^{\circ} \mathrm{C}$ with anti-BMP-2,

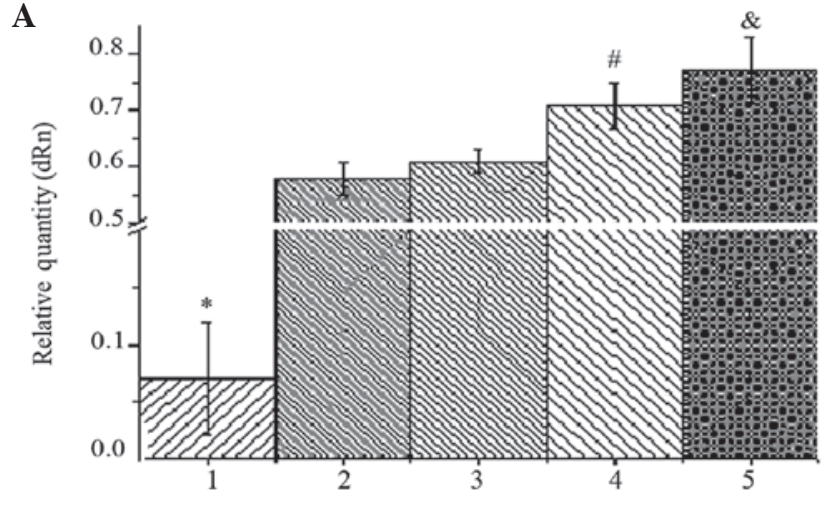

B
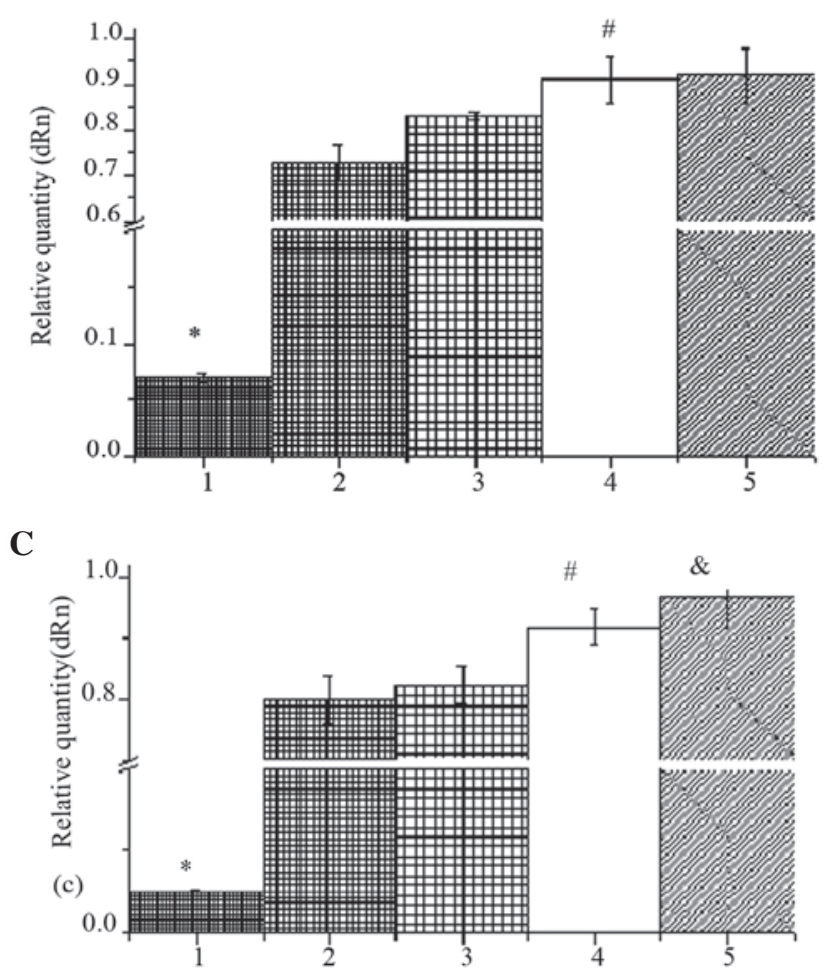

Figure 1. (A) BMP-2, (B) collagen I and (C) osteonectin mRNA content of osteoblasts detected by qPCR in 7 days. Bars: $1,1 \%$ aniline pentamer (AP)/poly(lactic-co-glycolic acid) (PLGA) alone; 2, PLGA with stimuli; $3,0.5 \%$ AP/PLGA with stimuli; $4,1 \%$ AP/PLGA with stimuli; and $5,5 \%$ AP/PLGA with stimuli. ${ }^{\&} \mathrm{P}<0.05$ when compared to 4 ; ${ }^{*} \mathrm{P}<0.05$ when compared to 2 and $3 ;{ }^{*} \mathrm{P}<0.05$ when compared to $2,3,4$ and $5 . \mathrm{n}=3$.

anti-Smad4, anti-collagen I, anti-osteonectin and anti-GAPDH monoclonal antibodies (Santa Cruz Biotechnology, Inc., Santa Cruz, CA, USA), diluted 5,000 times with phosphate-buffered saline (PBS) containing $1 \%$ bovine serum albumin and rinsed 4 times by $0.01 \mathrm{M}$ PBS, for 5 min each time. Color was developed using the Western Blotting DAB Testing kit (Beyotime). $\mathrm{X}$-ray film exposure was performed, followed by scanning and analyzing.

Origin 7.5 software was used for data processing and statistical analysis. Comparisons between groups were conducted with one-way ANOVA and Dunn's ad hoc test. Pearson's correlation coefficient was used to analyze the correlation between AP content and cell viability. $\mathrm{P}<0.05$ was considered to indicate a statistically significant difference. 
Table II. Cell viability of osteoblasts cultured on different materials (mean \pm standard deviation, $n=6$ ).

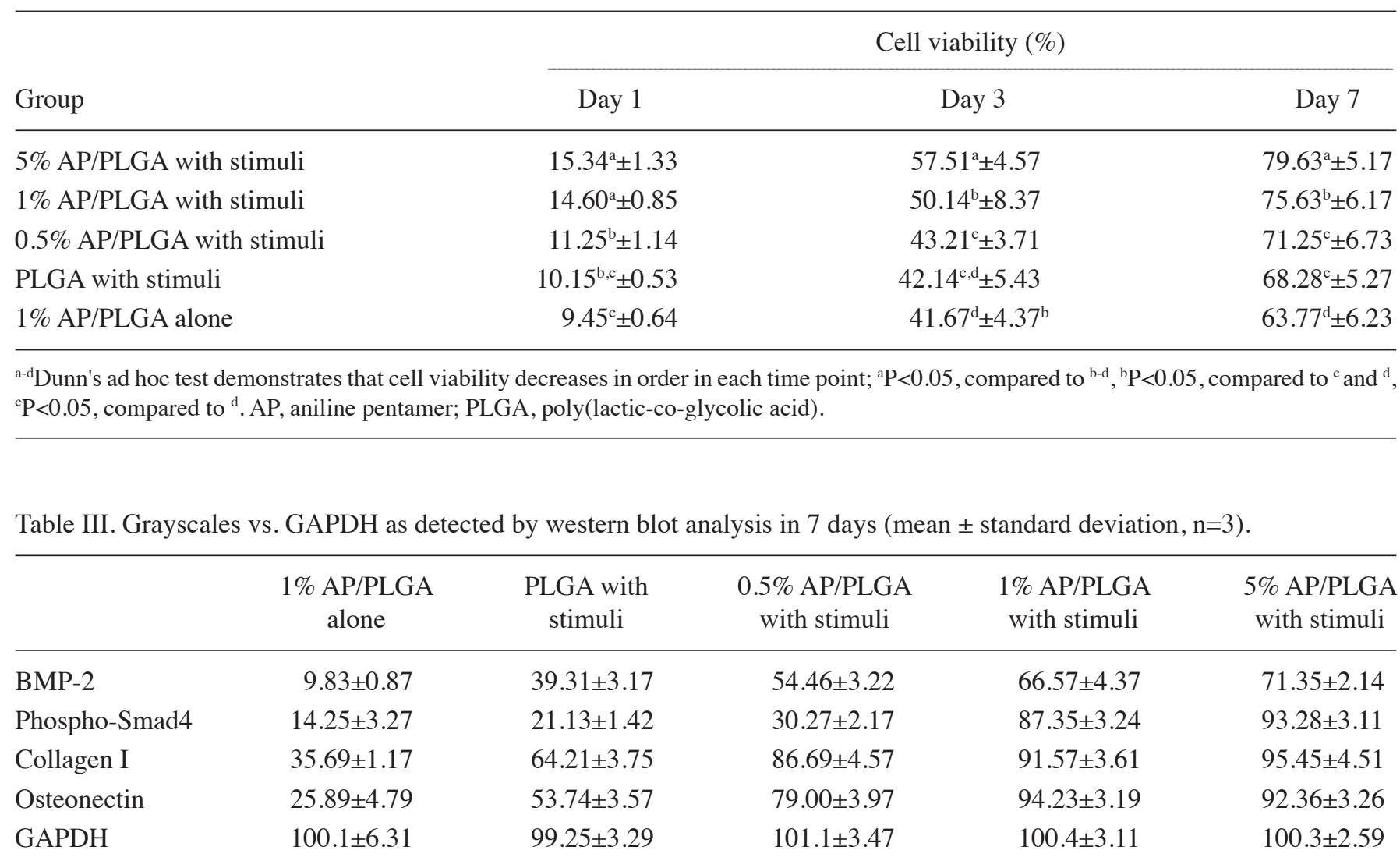

AP, aniline pentamer; PLGA, poly(lactic-co-glycolic acid).

\section{Results}

Preparation and characterization. UV-visible spectroscopy and cyclic voltammetry verified that the AP/PLGA nanocomposites were successfully prepared and conductive. The conductivity of AP/PLGA nanocomposites was significantly decreased compared to pure AP (data not shown).

Cell viability. All AP/PLGA nanocomposites in combination with electrical stimuli resulted in significantly higher cell viability compared to $1 \%$ AP/PLGA alone. The stimuli were effective in improving cell proliferation. Under electrical stimulation, 5 and 1\% AP/PLGA nanocomposites resulted in significantly higher cell viability compared to $0.5 \%$ AP/PLGA and PLGA. The ability of the stimuli to improve cell proliferation increased in proportion to the content of AP; however, there was no statistically significantly correlation (Pearson's correlation coefficients: $0.79,0.93$ and 0.89 ; Sig.: $0.22,0.06$ and 0.11 , respectively) (Table II).

Molecular expressions. Fig. 1 shows the expression of BMP-2, collagen I and osteonectin. The mRNA levels under electrical stimulation were significantly higher than those without stimulation. Under stimulation, the mRNA levels increased in proportion to the content of AP.

Fig. 2 shows the results of the western blot analysis of BMP-2, phospho-Smad4, collagen I and osteonectin. Their grayscales are listed in Table III. The protein levels under elec-

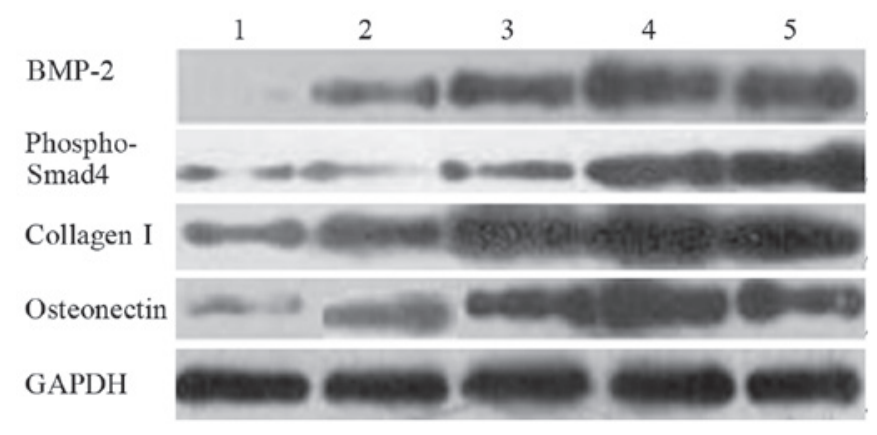

Figure 2. Western blot analysis. Lanes: 1, 1\% aniline pentamer (AP)/poly(lactic-co-glycolic acid) (PLGA) alone; 2, PLGA with stimuli; 3, 0.5\% AP/PLGA with stimuli; 4, 0.5\% AP/PLGA with stimuli; 5, 1 and 5\% AP/PLGA with stimuli.

trical stimulation were higher than those without stimulation. Under stimulation, the protein levels increased in proportion to the content of AP.

\section{Discussion}

The electric field and APs alone may improve the proliferation and osteogenic differentiation of osteoblasts. In the present study, rat-derived osteoblasts were cultured on AP/PLGA nanocomposite films, simultaneously exposed to electrical stimuli. The electrical stimulation combined with the AP implants 
significantly improved the proliferation and osteogenic differentiation of osteoblasts, compared to when each agent was used individually.

Various cell functions involve complicated signal transduction (16). The chemical signals are the main form of information transmission between cells; however, the intraand intercellular electrical signal transduction is an important contributing factor to cell behavior. Electric fields may directly promote the proliferation of osteoblasts. Two mechanisms may be involved in electric fields acting on osteoblasts. A previous study by de Barros Filho et al (2) reported that magnetic fields may directly promote DNA and protein synthesis in rat cells. Blank and Goodman (16) reported that pulsed electric fields may act directly on osteoblast DNA, affecting gene expression, thereby promoting the proliferation of osteoblasts. The electrical stimulation of osteoblasts may also be mediated by calcium ions or cAMP. There is evidence supporting this hypothesis. Normally, the gap junctional intercellular communication (GJIC), which is responsible for the transport of calcium ions or cAMP, is inhibited during normal tissue regeneration and cell proliferation; electric fields may inhibit the function of GJIC, thereby promoting cell proliferation (18).

Only cells that adhere to materials are able to migrate, differentiate and proliferate and the presence of a scaffold is necessary for tissue engineering and osteogenesis. APs exhibit higher solubility and conductivity. Therefore, in the present study, AP and PLGA were composited to produce electroactive biodegradable polymer nanocomposites, which appear promising for research or medical applications, including tissue engineering and regeneration of bone, cartilage or other tissues. As a result, electrical stimuli combined with AP/PLGA nanocomposite implants led to more positive outcomes compared to when used alone.

BMPs, particularly BMP-2, may induce mesenchymal stem cell differentiation into cartilage and bone, namely promote bone formation, by inducing formation and promoting proliferation of osteoblasts (19). Smad4 is a key signal involved in BMP expression, which promotes osteo-differentiation and -formation. Osteonectin, a non-collagenous matrix protein abundantly found in bone matrix, plays an important role in the process of mineralization by promoting mineral deposition in collagen (20). Osteonectin may promote extracellular matrix synthesis and increase the rate of bone matrix deposition (21). Collagen I is the scaffold for calcium deposition and cell adhesion. Collagen fibers comprise $~ 95 \%$ of the organic matter of the bone matrix and collagen I in particular comprises $\sim 90 \%$. Collagen I expression is initiated in the cell proliferation phase and reaches its peak in the matrix synthesis phase. In the proliferation phase, osteoblasts increase in number to form multilayers of cells, merge and secrete collagen I in order to mineralize and achieve the formation of bone nodules. Thereupon, BMP-2, Smad4, collagen I and osteonectin play important roles in the osteogenic process (22).

In the present study, qPCR and western blot analysis demonstrated that the expression of BMP-2, Smad4, collagen I and osteonectin was more upregulated by the combination of electrical stimuli and AP/PLGA, compared to each agent used alone, indicating that the combination may integrate and amplify the effects of the individual constituents.
In conclusion, AP and PLGA were successfully blended to create electroactive biodegradable nanocomposite AP/PLGA. The electrical stimuli, combined with the nanocomposites, may upregulate the expression levels of BMP-2, Smad4, collagen I and osteonectin and promote proliferation of rat-derived osteoblasts, compared to when each agent was used individually. The electroactive biodegradable nanocomposite AP/PLGA bears the potential for bone tissue engineering under electrical stimulation.

\section{References}

1. Ungethum M: Osteogenesis and bone growth. Modification by electrical and electromagnetic effects. MMW Munch Med Wochenschr 124: 621-622, 1982 (In German).

2. de Barros Filho TE, Rossi JD, Lage Lde A, Rodrigues CJ, de Oliveira AS, Pinto FC, dos Reis GM and Rodrigues Júnior AJ: Effect of electromagnetic fields on osteogenesis: an experimental study on rats. Rev Hosp Clin Fac Med Sao Paulo 47: 128-130, 1992 (In Portuguese).

3. Bekhite MM, Finkensieper A, Abou-Zaid FA, El-Shourbagy IK, Omar KM, Figulla HR, Sauer H and Wartenberg M: Static electromagnetic fields induce vasculogenesis and chondro-osteogenesis of mouse embryonic stem cells by reactive oxygen species-mediated up-regulation of vascular endothelial growth factor. Stem Cells Dev 19: 731-743, 2010.

4. Pietak AM, Reid JW, Stott MJ and Sayer M: Silicon substitution in the calcium phosphate bioceramics. Biomaterials 28: 4023-4032, 2007.

5. Wang L, Fan H, Zhang ZY, Lou AJ, Pei GX, Jiang S, Mu TW, Qin JJ, Chen SY and Jin D: Osteogenesis and angiogenesis of tissue-engineered bone constructed by prevascularized $\beta$-tricalcium phosphate scaffold and mesenchymal stem cells. Biomaterials 31: 9452-9461, 2010.

6. Gurkan UA, Kishore V, Condon KW, Bellido TM and Akkus O: A scaffold-free multicellular three-dimensional in vitro model of osteogenesis. Calcif Tissue Int 88: 388-401, 2011.

7. Zhao J, Han W, Chen H, Tu M, Huan S, Miao G, Zeng R, Wu H, Cha $\mathrm{Z}$ and Zhou C: Fabrication and in vivo osteogenesis of biomimetic poly(propylene carbonate) scaffold with nanofibrous chitosan network in macropores for bone tissue engineering. J Mater Sci Mater Med 23: 517-525, 2012.

8. Hu F, Chen S, Wang C, Yuan R, Xiang Y and Wang C: Multi-wall carbon nanotube-polyaniline biosensor based on lectin-carbohydrate affinity for ultrasensitive detection of Con A. Biosens Bioelectron 34: 202-207, 2012.

9. Plonska-Brzezinska ME, Mazurczyk J, Palys B, Breczko J, Lapinski A, Dubis AT and Echegoyen L: Preparation and characterization of composites that contain small carbon nano-onions and conducting polyaniline. Chemistry 18: 2600-2608, 2012.

10. Yang Y and Luan J: Synthesis, property characterization and photocatalytic activity of the novel composite polymer polyaniline $/ \mathrm{Bi}_{2} \mathrm{SnTiO}_{7}$. Molecules 17: 2752-2772, 2012.

11. Wong JY, Langer R and Ingber DE: Electrically conducting polymers can noninvasively control the shape and growth of mammalian cells. Proc Natl Acad Sci USA 91: 3201-3204, 1994.

12. Huang L, Hu J, Lang L, Wang X, Zhang P, Jing X, Wang X, Chen X, Lelkes PI, Macdiarmid AG and Wei Y: Synthesis and characterization of electroactive and biodegradable ABA block copolymer of polylactide and aniline pentamer. Biomaterials 28 : 1741-1751, 2007.

13. Huang J, Li Q, Wang Y, Wang Y, Dong L, Xie H and Xiong C: Self-suspended polyaniline doped with a protonic acid containing a polyethylene glycol segment. Chem Asian J 6: 2920-2924, 2011.

14. Feng W and Jia S: Rapamycin inhibits the invasive ability of thyroid cancer cells by down-regulating the expression of VEGF-C in vitro. Cell Biochem Funct 30: 487-491, 2012.

15. Galassi F, Kaman WE, Anssari Moin D, van der Horst J, Wismeijer D, Crielaard W, Laine ML, Veerman EC, Bikker FJ and Loos BG: Comparing culture, real-time PCR and fluorescence resonance energy transfer technology for detection of Porphyromonas gingivalis in patients with or without peri-implant infections. J Periodontal Res 47: 616-625, 2012.

16. Blank M and Goodman R: Do electromagnetic fields interact directly with DNA? Bioelectromagnetics 18: 111-115, 1997. 
17. Huang L, Zhuang X, Hu J, Lang L, Zhang P, Wang Y, Chen X, Wei Y and Jing X: Synthesis of biodegradable and electroactive multiblock polylactide and aniline pentamer copolymer for tissue engineering applications. Biomacromolecules 9: 850-858, 2008.

18. Li CM, Chiang H, Fu YD, Lu DQ and Shao J: Exposure to 50-Hz electromagnetic fields: effects of time and field strength on GAP junctional intercellular communications. Electromagn Biol Med 18: 249-256, 1999

19. Kim SS, Park MS, Jeon O, Choi CY and Kim BS: Poly(lactide-co-glycolide)/hydroxyapatite composite scaffolds for bone tissue engineering. Biomaterials 27: 1399-1409, 2006.
20. Termine JD, Kleinman HK, Whitson SW, Conn KM, McGarvey ML and Martin GR: Osteonectin, a bone-specific protein linking mineral to collagen. Cell 26: 99-105, 1981.

21. Ribeiro N, Sousa SR and Monteiro FJ: Influence of crystallite size of nanophased hydroxyapatite on fibronectin and osteonectin adsorption and on MC3T3-E1 osteoblast adhesion and morphology. J Colloid Interface Sci 351: 398-406, 2010.

22. Lopez JM, Balemans W, Piters E, van Hul W and González G: Genetic analysis and effect of triiodothyronine and prednisone trial on bone turnover in a patient with craniotubular hyperostosis. Bone 43: 405-409, 2008. 